\title{
Safety and Effectiveness of Varenicline in Korean Smokers: A Nationwide Post-Marketing Surveillance Study
}

\author{
Minsu Ock $\mathbb{D}^{1}$, Ji Soo $\mathrm{Shin}^{2}$, Seung Won Ra $\mathbb{D}^{3}$ \\ 'Department of Preventive Medicine, Ulsan University Hospital, University of Ulsan College of Medicine, Ulsan, South Korea; ${ }^{2}$ Medical Affairs, Pfizer \\ Korea, Seoul, South Korea; ${ }^{3}$ Department of Internal Medicine, Ulsan University Hospital, University of Ulsan College of Medicine, Ulsan, South Korea \\ Correspondence: Seung Won Ra, Department of Internal Medicine, Ulsan University Hospital, University of Ulsan College of Medicine, 877 \\ Bangeojinsunhwan-doro, Dong-gu, Ulsan, 44033, South Korea, Tel +82 52250 8857, Fax +82 52 250 7048, Email docra@uuh.ulsan.kr
}

\begin{abstract}
Purpose: Varenicline has demonstrated its safety and efficacy in Western studies including $<3 \%$ of Asian participants. This prospective multi-center observational study investigated the safety and effectiveness of varenicline in Korean smokers.

Patients and Methods: Smokers prescribed varenicline for the first time were enrolled from 252 medical institutions. Investigators recorded and graded all adverse events (AEs). To assess the effectiveness of varenicline, the 7-day point prevalence (PP) of smoking cessation was evaluated at the four visits during the 12-week treatment course. Rates of AE incidence and smoking cessation were analyzed using Chi-squared test or Fisher's exact test. This study is registered with ClinicalTrials.gov, number NCT00483002.

Results: A total of 3719 and 3700 study subjects were included in the safety and effectiveness analyses, respectively. Overall, 346 (9.3\%) subjects experienced 471 AEs: 358 mild, 97 moderate, 9 severe, and 7 serious. The most frequent AEs were nausea (5.1\%), dyspepsia $(0.8 \%)$, abnormal dreams $(0.8 \%)$, insomnia $(0.8 \%)$, and headache $(0.7 \%)$. Among the subjects with AEs, 73 subjects discontinued treatment, of which 68 were due to AE occurrence. The 7-day PP of smoking cessation at weeks 1-2, 3-6, 7-10, and $\geq$ week 11 were 51.7\% (387/749), 59.6\% (1740/2922), 73.3\% (1114/1520), and 77.0\% (1116/1449), respectively ( $\mathrm{p}$ for trend $=0.023)$. Comorbidities and allergies were associated with a higher incidence of AEs and lower smoking cessation rate $(p<0.05)$. Younger subjects, infrequent alcohol drinkers, and lighter smokers showed a higher smoking cessation rate $(\mathrm{p}<0.05)$.

Conclusion: Varenicline in adult Korean smokers was well tolerated and effective as a smoking cessation aid in routine clinical practice.
\end{abstract}

Keywords: adverse drug events, smoking cessation, Asia, varenicline, product surveillance

\section{Plain Language Summary}

Can varenicline be used safely and effectively in Korean smokers?

In this largest observational study in Asia, we evaluated the tolerability and effectiveness of varenicline in routine clinical setting. Nausea was the most common adverse event (AE); however, most reported cases were of mild or moderate intensity. Other commonly occurring side effects included dyspepsia, abnormal dreams, insomnia, and headache. AE incidence doubled in smokers with allergy/ comorbidity history. Neuropsychiatric adverse events such as depression or anxiety were rarely documented although no subjects were excluded for related criteria. Smokers with young age, less frequent drinking, less smoking amount, few attempts to quit smoking, and without concomitant use of medications were more likely to quit smoking. Varenicline's effectiveness in smoking cessation as assessed by the never-smoking rate in the last 7 days was $67.9 \%$, which was higher than that from other interventional or observational studies. Our study significantly contributes to the safety and effectiveness of varenicline in Asian population.

\section{Introduction}

Smoking causes major diseases like cardiovascular disease, chronic obstructive pulmonary disease, and lung cancer. ${ }^{1}$ Globally, $25.0 \%$ males and 5.4\% females smoke daily, and $11.5 \%$ global deaths in 2015 were attributed to smoking. ${ }^{2}$ 
Despite the well-known health risks associated with tobacco use, smoking is highly prevalent in South Korea, particularly in males. According to the Korea National Health and Nutrition Examination Survey report from 2008 to 2011 , the smoking rate based on urinary cotinine results was $52.7 \%$ and $15.4 \%$ for Korean adult males and females, respectively, and surprisingly, the female result was more than twice the self-reported smoking rate $(6.8 \%){ }^{3}$

Smoking can be attributed to nicotine addiction; therefore, aggressive addiction treatment using smoking cessation medications may be implemented for a better outcome. ${ }^{4}$ Among various smoking cessation aids, varenicline has proved its efficacy for smoking cessation and is often recommended to use by clinicians. ${ }^{5,6}$ Varenicline has a high affinity for the predominant brain nicotinic receptor, $\alpha 4 \beta 2$, responsible for mediating the reinforcing properties of nicotine in animal models. ${ }^{7}$ As a partial agonist, varenicline has advantages over currently available therapies. The agonist property may reduce cravings and withdrawal symptoms, whereas the antagonist property may reduce the reward experienced by those who smoke during therapy, thereby reducing the likelihood of relapse. ${ }^{4}$

There were several concerns regarding the adverse events (AEs) of varenicline. Nausea, headache, insomnia, constipation, and abdominal pain are common AEs regardless of ethnicity. ${ }^{8-12}$ Although serious neuropsychiatric AEs are anecdotally reported, no causal relationship has been reported in clinical trials or large cohort studies. ${ }^{11,13,14}$ As these studies were conducted in Western countries with a limited number of Asian participants, and AEs can be diverse and unpredictable among different ethnicities, caution is needed when considering the safety outcomes in Asian populations. Recently, there has been a safety issue related to potential carcinogenicity in the varenicline products. Therefore, a long-term and large-scale post-marketing surveillance (PMS) for varenicline that monitors both common and unrecognized AEs ${ }^{15}$ may provide evidence in these regards. In addition, information on the effectiveness of varenicline collected from PMS performed in a real-world setting will be useful in evaluating the risk-benefit profile of the drug in Korean population.

This is a nationwide PMS study wherein varenicline was administered to 3719 Korean smokers over a 12-week period. We aimed to evaluate the safety and effectiveness of varenicline in usual medical practice in South Korea.

\section{Materials and Methods}

\section{Study Design and Population}

This prospective non-interventional monitoring study was conducted at 252 medical institutions from 2007 to 2013 according to routine clinical practice of physicians from different departments, including cardiology, pulmonology, endocrinology, family medicine, and general practitioners' clinics. The study enrolled subjects aged $\geq 18$ years, who were prescribed varenicline for the first time. They were excluded from the study if they did not meet the prescribing criteria for varenicline or disagreed to participate in the study. There was no systemic assignment for treatment, and the medication was prescribed at the discretion of physicians, independent of study participation.

\section{Treatment and Follow Up}

Investigators and study subjects made a shared decision to use varenicline, and the drug was dispensed with product information, followed by the physician's prescription. The first prescription visit was considered the baseline visit (week 0 ). Subjects decided on a quit date and started treatment for up to 2 weeks before this date. Subjects were advised to take varenicline for 12 weeks: $0.5 \mathrm{mg}$ of varenicline once daily for the first 3 days, titrated to $0.5 \mathrm{mg}$ twice daily on days $4-7$, then $1 \mathrm{mg}$ twice daily from day 8 to the end of the 12-week treatment phase. The start and end dates of treatment and the daily dose and frequency were recorded. If subjects did not complete the study, the reason for discontinuation was identified and classified as one of the following: "AE," "lack of efficacy," "success in smoking cessation," "lost to follow up," or "others." Physicians recorded the concomitant medications administered continuously during or after enrollment.

\section{Safety and Effectiveness Evaluation}

AE was defined as any untoward medical occurrence in a study subject taking varenicline. All AEs were recorded from the time the subject has taken at least one dose of varenicline through last subject visit. The investigators obtained and recorded all observed or self-reported AEs and their severity (mild [does not interfere with subject's usual function], moderate [interferes to some extent with subject's usual function], or severe [interferes significantly with subject's usual 
function]) on the case report forms along with their opinions on the causal relationship with the study drug. For all AEs, the investigators obtained adequate information to determine whether it met the criteria for classification as a serious adverse event (SAE). SAEs were classified as any untoward medical occurrence at any dose that resulted in death, was life-threatening (immediate risk of death), required hospitalization, or resulted in significant disability. SAEs were reported from the time that subject provides informed consent through and including 28 calendar days after the last administration of the study drug. Any SAE occurring after the reporting period was also reported if suspected of causal relationship to varenicline.

Effectiveness was measured by the 7-day point prevalence (PP) of smoking cessation, which was evaluated on visits 1 (weeks 1-2), 2 (weeks 3-6), 3 (weeks 7-10), and 4 (on or beyond week 11) during the 12-week treatment course. Subjects were considered to have quit when they answered "No" to both of the following two questions: "Have you smoked any cigarettes (even a puff) in the last 7 days?" and "Have you used any other product containing nicotine in the last 7 days?" Examples of products containing nicotine were nicotine patches/gums/nasal sprays/inhalers/tablets, snuff, pipes, and cigars.

\section{Statistical Analysis}

Demographic and baseline characteristics were reported as descriptive statistics. To describe continuous variables, mean \pm standard deviation (SD) and range were presented. For categorical variables, frequency and percentage were used. $\mathrm{AE}$ incidence and smoking cessation rates with $95 \%$ confidence interval (CI) were estimated using Chi-squared test or Fisher's exact test. Data with missing values were excluded. Statistical analyses were performed using SAS Software version 9.1. All statistical tests were two-sided with a significance threshold of 0.05 .

\section{Ethical Approval}

The final protocol, amendments, and informed consent form were reviewed and approved by the institutional review board(s) and/or independent ethics committee(s) at each investigational center (Supplementary Table 1). The study conformed to the European Medicines Agency Guidelines for Good Clinical Practice. The investigators explained the nature, purpose, and duration of the study to the subjects and informed them that they could withdraw from the study at any time for any reason. After providing sufficient time to consider the implications of the study, written informed consents were obtained from subjects before study participation. This study is registered with ClinicalTrials.gov, number NCT00483002.

\section{Results}

\section{Study Subjects' Characteristics}

Figure 1 presents the study subject selection. Overall, 3722 subjects were enrolled, and 3719 who received at least one dose of the study drug were included in the safety analysis. Subsequently, 19 were excluded because no effectiveness assessments were available (Figure 1), and 3700 were included in the effectiveness analysis. Of these, 1165 (31.5\%) completed our study and $2535(68.5 \%)$ dropped out before the end of the 12-week treatment period. Discontinuations were attributed to either AEs (68, 2.7\%), lack of efficacy (555, 21.9\%), smoking cessation (935, 36.9\%), failure to follow up (943, 37.2\%), or other reasons (eg, patient refusal, expensive cost).

The demographic characteristics of subjects in the safety analysis are provided in Table 1. Overall, $90.9 \%$ subjects were male with a mean age ( \pm SD) of $49.4 \pm 11.7$ years. A high proportion of Korean smokers $(59.3 \%)$ consumed alcohol once or twice per week. Furthermore, 1903 (51.2\%) started smoking at the age of 21-30 years and 1594 (42.9\%) at the age of 15-20 years. Most subjects $(2118,57.0 \%)$ smoked an average of one to two packs of cigarettes per day, and 1413 (38.1\%) had previously attempted to stop smoking, at least two to four times. Overall, 48 subjects $(1.3 \%)$ had a history of allergy. 1612 (43.3\%) subjects had $\geq 1$ comorbidity, including hypertension (714, 44.3\%), chronic obstructive pulmonary disease or other pulmonary diseases $(500,31.0 \%)$, diabetes mellitus $(414,25.7 \%)$, dyslipidemia $(331,20.5 \%)$, coronary heart disease $(120,7.4 \%)$, stroke or transient ischemic attack $(35,2.2 \%)$, and others $(21.5 \%)$ like gastrointestinal disease, 


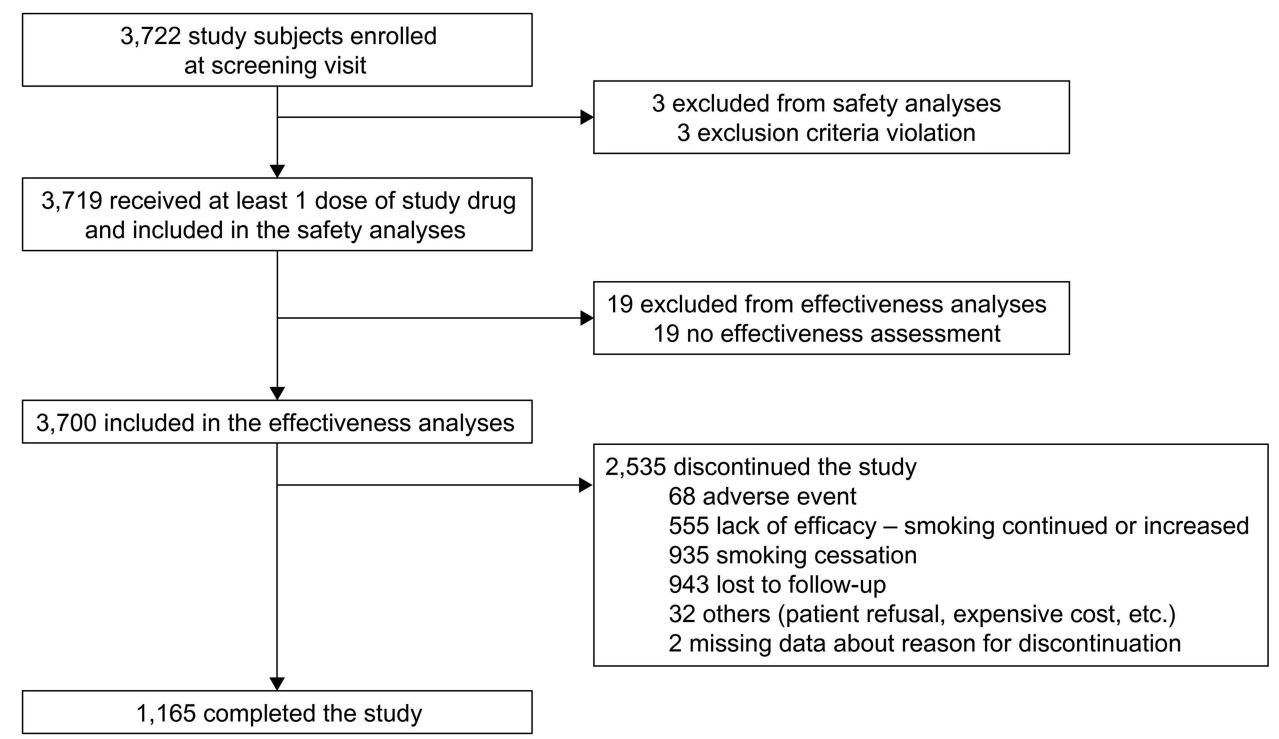

Figure I Flow chart for selection of study subjects.

osteoporosis, depression, cancer, liver disease, and thyroid disease. Furthermore, $1486(40.0 \%)$ had been taking concomitant medications.

\section{Adverse Events}

The mean total administration period of varenicline was $52.9 \pm 32.3$ days, and the mean daily dose and mean total dose of varenicline were $1.68 \pm 0.3$ and $94.2 \pm 63.5 \mathrm{mg}$, respectively, in the safety analysis population (Supplementary Table 2). Of the 3719 subjects, 346 (9.3\%) experienced at least one AE, with 471 AEs (464 AEs and 7 SAEs) reported overall.

Table 2 summarizes AEs (excluding SAEs) with their severity information. Of the 464 AEs, 358, 97, and 9 were mild, moderate, and severe, respectively. The most frequently reported AEs were nausea (5.1\%), dyspepsia (0.8\%), abnormal dreams $(0.8 \%)$, insomnia (0.8\%), and headache (0.7\%). Psychiatric AEs occurred in $2.1 \%$ of study subjects, including 8 $(0.2 \%)$ events of depression. Four subjects experienced seven SAEs, including syncope, insomnia, anxiety, abnormal thinking, pneumonia, cerebral artery stenosis, and vestibular neuronitis. Aggravation of lung neoplasm was reported as an AE by one subject (Supplementary Table 3). For long-term results, 6 (42.9\%) subjects had experienced AEs among 14 subjects who took varenicline for 24 weeks or longer during the study period.

A history of allergy or comorbidity was associated with a higher incidence of AE (history of allergy: $20.8 \% \mathrm{vs} 9.2 \%$, $\mathrm{p}=0.011$; comorbidity: $12.8 \%$ vs $6.6 \%, \mathrm{p}=0.0005$ ) (Table 3). AEs were more frequently reported in subjects taking concomitant medications than those who were not $(13.1 \%$ vs $6.8 \%, \mathrm{p}<0.001)$. Significantly more AEs were reported from the group with a mean daily dose of varenicline $(\mathrm{mg})$ of $0.5-<1.0$ or $1.0-<1.5$ than the group taking a dose of 1.5$<2.0(\mathrm{p}=0.027)$. The total varenicline administration period (weeks) was not associated with AE incidence. Among all subjects with AEs, 73 discontinued treatment, wherein 68 (19.7\%) were due to AE occurrence. Dose reduction due to AE or death was not reported from this study.

\section{Seven-Day Point Prevalence of Smoking Cessation}

As shown in Figure 2, the 7-day PP of smoking cessation on visit week 1-2, 3-6, 7-10, and $\geq$ week 11 was 51.7\% (387/749), $59.6 \%$ (1740/2922), 73.3\% (1114/1520), and 77.0\% (1116/1449), respectively ( $\mathrm{p}$ for trend $=0.023)$. When the smoking cessation rate evaluated by 7-day PP at the last observed study visit was analyzed according to the baseline characteristics and treatment pattern (Table 4), younger subjects exhibited significantly higher smoking cessation rate than older subjects $(\mathrm{p}=0.019)$; however, age at smoking initiation was not associated with smoking cessation $(p=0.085)$. Subjects who drank alcohol more than thrice per week had a lower smoking cessation rate compared to those who drank less $(\mathrm{p}<0.001)$. Lower smoking cessation rate was observed in subjects who smoked more than one pack per day compared to those who smoked less $(\mathrm{p}<0.001)$. Subjects who had 
Table I Baseline Characteristics of Study Subjects

\begin{tabular}{|c|c|c|}
\hline & & Number of Subjects (\%) \\
\hline \multirow[t]{2}{*}{ Sex } & Male & 3379 (90.9) \\
\hline & Female & $340(9.1)$ \\
\hline \multirow[t]{4}{*}{ Age } & $18-44$ & I264 (34.0) \\
\hline & $45-64$ & $2031(54.6)$ \\
\hline & $\geq 65$ & $424(11.4)$ \\
\hline & Mean \pm SD (range) & $49.4 \pm 11.7(18-86)$ \\
\hline \multirow[t]{3}{*}{ Number of drinks per week ${ }^{\mathrm{a}}$} & None & $733(19.7)$ \\
\hline & $1-2$ & $2205(59.3)$ \\
\hline & $\geq 3$ & $780(21.0)$ \\
\hline \multirow[t]{4}{*}{ Age at smoking initiation ${ }^{\mathrm{a}}$ (years) } & $<15$ & $121(3.3)$ \\
\hline & $15-20$ & 1594 (42.9) \\
\hline & $21-30$ & 1903 (5I.2) \\
\hline & Others & $100(2.7)$ \\
\hline \multirow{4}{*}{$\begin{array}{l}\text { Average smoking amount per day } \\
(\text { packs })^{\mathrm{a}}\end{array}$} & $<1$ & $1204(32.4)$ \\
\hline & $1-2$ & $2118(57.0)$ \\
\hline & $2-3$ & $379(10.2)$ \\
\hline & Others & $17(0.5)$ \\
\hline \multirow[t]{4}{*}{ Number of attempts to quit smoking ${ }^{\mathrm{b}}$} & None & $975(26.3)$ \\
\hline & Once & $729(19.6)$ \\
\hline & $2-4$ times & $1413(38.1)$ \\
\hline & $\geq 5$ times & $596(16.1)$ \\
\hline \multirow[t]{2}{*}{ Allergy history } & No & $367 \mid(98.7)$ \\
\hline & Yes & $48(1.3)$ \\
\hline \multirow[t]{2}{*}{ Comorbidity $^{c}$} & No & $2107(56.7)$ \\
\hline & Yes & $1612(43.3)$ \\
\hline \multirow[t]{2}{*}{ Concomitant medication } & No & $2233(60.0)$ \\
\hline & Yes & $1486(40.0)$ \\
\hline
\end{tabular}

Notes: aOne missing. Among "Others" for age at smoking initiation, 83 subjects started smoking after 30 years of age, while 17 provided no information. Among "Others" for average smoking amount per day, 16 subjects smoked three packs or more per day on average, while one subject provided no information; 'Six missing; 'Some subjects had $\geq 1$ concomitant disease: 714 cases of hypertension (44.3\%), 500 cases of chronic obstructive pulmonary disease or other pulmonary diseases (31.0\%), 414 cases of diabetes mellitus (25.7\%), 331 cases of dyslipidemia (20.5\%), I 20 cases of coronary heart disease (7.4\%), 35 cases of stroke or transient ischemic attack (2.2\%), and others (2I.5\%), including gastrointestinal disease, osteoporosis, depression, cancer, liver disease, and thyroid disease.

previously attempted to quit smoking more than five times were the least successful in smoking cessation among groups of the number of attempts to quit smoking with borderline significance $(64.9 \% ; \mathrm{p}=0.050)$. Allergy history, comorbidity, and concomitant medication were associated with lower smoking cessation rates $(\mathrm{p}<0.01)$. Smoking cessation rate significantly 
Table 2 Summary of Adverse Events with Occurrence in 5 Subjects or More

\begin{tabular}{|c|c|c|c|c|c|}
\hline \multirow[t]{2}{*}{ Events } & \multicolumn{2}{|c|}{ Number of $A E s^{a}$} & \multicolumn{3}{|c|}{ Severity } \\
\hline & $\mathbf{N}$ & $\%$ & Mild & Moderate & Severe \\
\hline Gastrointestinal disorders & 239 & 6.4 & 195 & 42 & 4 \\
\hline Abdominal pain & 13 & 0.3 & 10 & 3 & 0 \\
\hline Constipation & 12 & 0.3 & 9 & 3 & 0 \\
\hline Dyspepsia & 30 & 0.8 & 21 & 8 & I \\
\hline Nausea & 191 & 5.1 & 157 & 31 & 3 \\
\hline Vomiting & 9 & 0.2 & 7 & 2 & 0 \\
\hline Psychiatric disorders & 78 & 2.1 & 63 & 17 & 0 \\
\hline Abnormal dreams & 30 & 0.8 & 25 & 5 & 0 \\
\hline Depression & 8 & 0.2 & 4 & 4 & 0 \\
\hline Insomnia & 30 & 0.8 & 28 & 2 & 0 \\
\hline Nightmare & 6 & 0.2 & 3 & 3 & 0 \\
\hline Sleep disorder & 14 & 0.4 & 11 & 3 & 0 \\
\hline Nervous system disorders & 42 & I.I & 32 & 7 & 3 \\
\hline Dizziness & 5 & 0.1 & 3 & 1 & I \\
\hline Headache & 26 & 0.7 & 20 & 5 & I \\
\hline Somnolence & 9 & 0.2 & 8 & I & 0 \\
\hline Metabolism and nutrition disorders & 9 & 0.2 & 7 & 2 & 0 \\
\hline Increased appetite & 6 & 0.2 & 5 & I & 0 \\
\hline Skin and subcutaneous tissue disorders & 8 & 0.2 & 5 & 3 & 0 \\
\hline Pruritus & 5 & 0.1 & 3 & 2 & 0 \\
\hline Others & 22 & 0.6 & 15 & 7 & 0 \\
\hline Appetite decreased/anorexia & 7 & 0.2 & 5 & 2 & 0 \\
\hline Fatigue/malaise/adynamia & 17 & 0.5 & 12 & 5 & 0 \\
\hline Total & 464 & 100.0 & 358 & 97 & 9 \\
\hline
\end{tabular}

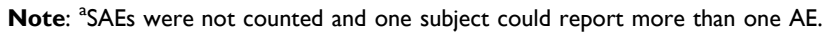
Abbreviations: AE, adverse event; SAE, serious adverse event.

increased with increasing mean total administration period of varenicline $(\mathrm{p}<0.0001)$. Although the quit rate was significantly different between groups of mean daily dose of varenicline, no specific trend was observed.

The smoking cessation rate at the last observed study visit was significantly higher in subjects who completed the study than those who did not $(83.4 \%$ vs $60.8 \% ; p<0.001)$ (Table 5). The most common reason for discontinuation in the smoking cessation group was an early achievement of smoking cessation (60.4\%) followed by loss to follow up (37.5\%). In the continued smoking group, lack of efficacy (ie, continued smoking or increased smoking, 55.6\%) was the most common reason for dropout followed by loss to follow up (38.1\%). In both groups, AE was not the major determinant for dropouts. 
Table 3 Incidence of Adverse Events by Baseline Characteristics and Treatment Pattern

\begin{tabular}{|c|c|c|c|c|c|c|}
\hline & & $\begin{array}{l}\text { Number of } \\
\text { Subjects (\%) }\end{array}$ & $\begin{array}{l}95 \% \text { Cl (Lower, } \\
\text { Upper) }\end{array}$ & $\begin{array}{l}\text { Number of } \\
\text { Events }\end{array}$ & Total $\mathbf{N}$ & p value \\
\hline \multirow[t]{2}{*}{ Sex } & Male & $319(9.4)$ & $(8.5,10.4)$ & 431 & 3379 & \multirow[t]{2}{*}{0.364} \\
\hline & Female & $27(7.9)$ & $(5.1,10.8)$ & 40 & 340 & \\
\hline \multirow[t]{6}{*}{ Age (years) } & $<30$ & $20(13.3)$ & $(7.9,18.8)$ & 24 & 150 & \multirow[t]{6}{*}{0.460} \\
\hline & $30-39$ & $64(9.9)$ & $(7.6,12.2)$ & 95 & 648 & \\
\hline & $40-49$ & $95(8.6)$ & $(6.9,10.2)$ & 127 & IIII & \\
\hline & $50-59$ & $98(9.2)$ & $(7.5,10.9)$ & 132 & 1067 & \\
\hline & $60-69$ & $56(9.8)$ & $(7.4,12.2)$ & 75 & 572 & \\
\hline & $\geq 70$ & $13(7.6)$ & $(3.6,11.6)$ & 18 & 171 & \\
\hline \multirow{3}{*}{$\begin{array}{l}\text { Alcohol use (drinking frequency } \\
\text { per week) }\end{array}$} & None & $76(10.4)$ & $(8.2,12.6)$ & 115 & 733 & \multirow[t]{3}{*}{0.400} \\
\hline & $1-2$ & $194(8.8)$ & $(7.6,10.0)$ & 254 & 2205 & \\
\hline & $\geq 3$ & $76(9.7)$ & $(7.7, \mid 1.8)$ & 102 & 780 & \\
\hline \multirow{4}{*}{$\begin{array}{l}\text { Average smoking amount per day } \\
\text { (pack) }\end{array}$} & $<1$ & $126(10.5)$ & $(8.7,12.2)$ & 174 & 1204 & \multirow[t]{4}{*}{0.215} \\
\hline & $\mathrm{I}-2$ & 185 (8.7) & $(7.5,9.9)$ & 251 & 2118 & \\
\hline & $2-3$ & $32(8.4)$ & $(5.6, \mid I .2)$ & 43 & 379 & \\
\hline & Others & $3(17.7)$ & $(0.0,35.8)$ & 3 & 17 & \\
\hline \multirow[t]{2}{*}{ Allergy history } & No & $336(9.2)$ & $(8.2,10.1)$ & 455 & 3671 & \multirow[t]{2}{*}{0.011} \\
\hline & Yes & $10(20.8)$ & $(9.3,32.3)$ & 16 & 48 & \\
\hline \multirow[t]{9}{*}{ Comorbidity } & No & $139(6.6)$ & $(5.5,7.7)$ & 188 & 2107 & \multirow[t]{2}{*}{0.0005} \\
\hline & Yes & $207(12.8)$ & $(11.2,14.5)$ & 283 & 1612 & \\
\hline & $\mathrm{CHD}$ & $10(8.3)$ & $(11.2,14.5)$ & 14 & 120 & \\
\hline & Diabetes mellitus & $4 \mid(9.9)$ & $(3.34,13.3)$ & 54 & 414 & \\
\hline & $\begin{array}{l}\text { COPD and other } \\
\text { pulmonary disease }\end{array}$ & $73(14.6)$ & $(7.0,12.8)$ & 103 & 500 & \\
\hline & Hypertension & 83 (1I.6) & $(11.5,17.7)$ & 108 & 714 & \\
\hline & Dyslipidemia & $42(12.7)$ & $(9.1,16.3)$ & 58 & 331 & \\
\hline & Stoke or TIA & $0(0.0)$ & $(0.0,0.0)$ & 0 & 35 & \\
\hline & Others & $53(15.3)$ & $(11.5,19.1)$ & 82 & 347 & \\
\hline \multirow[t]{2}{*}{ Concomitant medication } & No & $|5|(6.8)$ & $(5.7,7.8)$ & 208 & 2233 & \multirow[t]{2}{*}{$<0.001$} \\
\hline & Yes & $195(13.1)$ & $(I I .4,14.8)$ & 263 & 1486 & \\
\hline \multirow{3}{*}{$\begin{array}{l}\text { Total administration period of } \\
\text { varenicline (weeks) }\end{array}$} & $<4$ & $76(8.8)$ & $(6.9,10.7)$ & 119 & 865 & \multirow[t]{3}{*}{0.088} \\
\hline & $4-8$ & $97(8.1)$ & $(6.6,9.7)$ & 123 & 1197 & \\
\hline & $>8$ & $173(10.4)$ & $(9.0,11.9)$ & 229 & 1657 & \\
\hline
\end{tabular}


Table 3 (Continued).

\begin{tabular}{|l|l|l|l|l|l|l|}
\hline \multicolumn{2}{|c|}{} & $\begin{array}{l}\text { Number of } \\
\text { Subjects (\%) }\end{array}$ & $\begin{array}{l}\text { 95\% Cl (Lower, } \\
\text { Upper) }\end{array}$ & $\begin{array}{l}\text { Number of } \\
\text { Events }\end{array}$ & $\begin{array}{l}\text { Total N } \\
\text { P value }\end{array}$ \\
\hline \multirow{2}{*}{$\begin{array}{l}\text { Mean daily dose of varenicline } \\
(\mathrm{mg})\end{array}$} & $0.5-<1.0$ & $30(12.8)$ & $(8.5,17.0)$ & 49 & 235 & 0.027 \\
\cline { 2 - 7 } & $1.0-<1.5$ & $52(12.1)$ & $(9.0,15.1)$ & 75 & 431 \\
\cline { 2 - 7 } & $1.5-<2.0$ & $256(8.6)$ & $(7.6,9.6)$ & 337 & 2978 \\
\cline { 2 - 7 } & $\geq 2.0$ & $8(10.7)$ & $(3.7,17.7)$ & 10 & 75 \\
\hline \multirow{2}{*}{ Total } & $346(9.3)$ & $(8.4,10.2)$ & 471 & 3719 \\
\hline
\end{tabular}

Notes: ${ }^{a} \mathrm{p}$ value was calculated using the Chi-squared test.

Abbreviations: $\mathrm{Cl}$, confidence interval; $\mathrm{N}$, number of study subjects; $\mathrm{CHD}$, congestive heart disease; COPD, chronic obstructive pulmonary disease; TIA, transient ischemic attack.

\section{Discussion}

Overall, 3719 study subjects were treated with varenicline and analyzed for safety, and 3700 were analyzed for effectiveness. To our knowledge, this is the largest observational study to investigate the safety and effectiveness of varenicline in Asian smokers.

To ensure varenicline is properly prescribed to all who may benefit, it is necessary to address misconcerns regarding its AEs. Following reports of varenicline linked to mental health issues, the US Food and Drug Administration once mandated a boxed label warning for varenicline regarding neuropsychiatric or cardiovascular AEs. ${ }^{16,17}$ However, varenicline could provide evidence of its safety and tolerability. ${ }^{11,14,18-21}$ The EAGLES study, a large multi-national randomized controlled study, reported no significant increase in neuropsychiatric AEs due to varenicline in patients with and without psychiatric disorders. ${ }^{11}$ A meta-analysis of 39 randomized controlled trials showed no evidence of increased

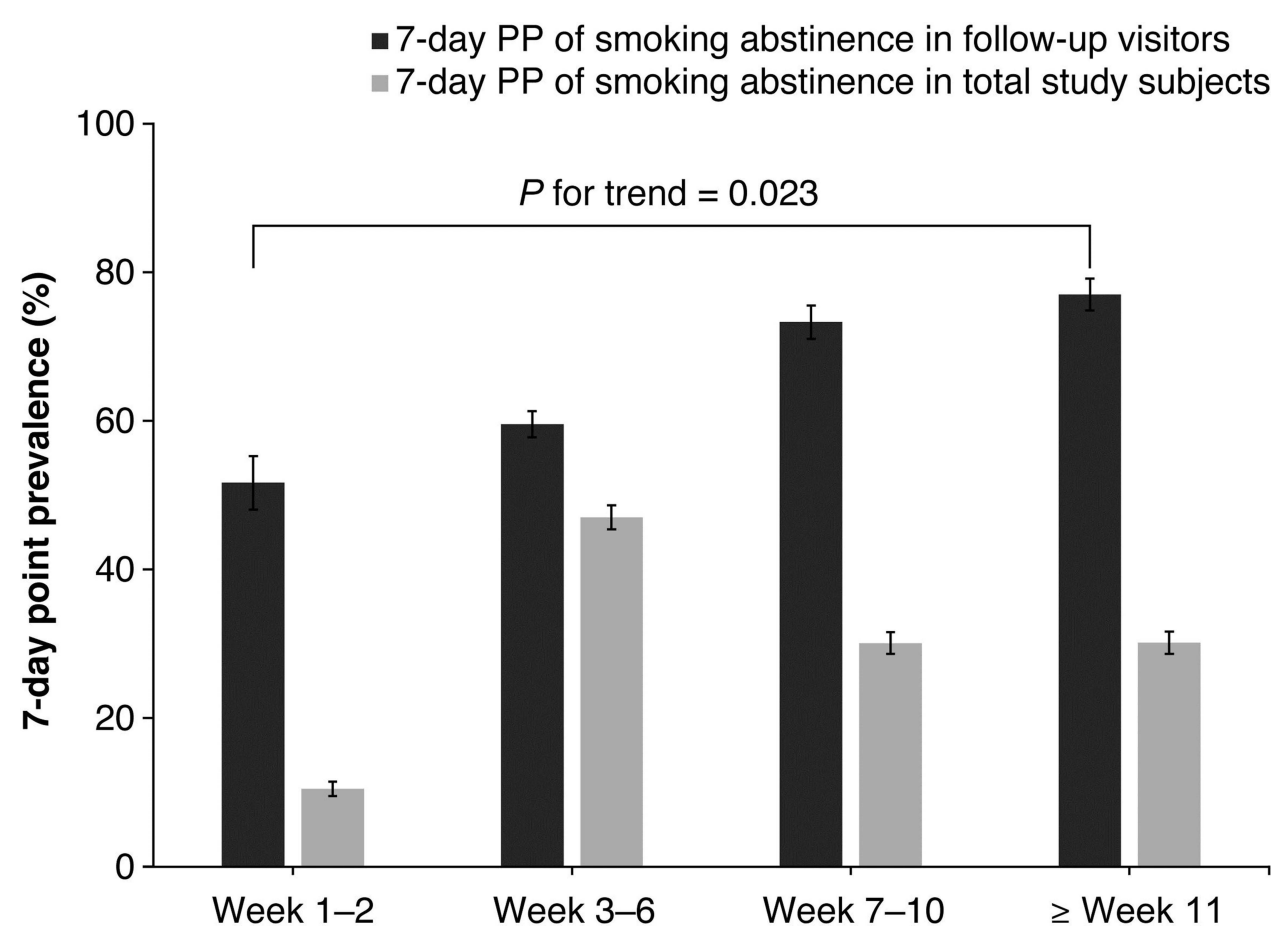

Figure 2 Seven-day point prevalence (PP) of smoking abstinence. Error bars indicate $95 \%$ confidence intervals. The 7-day PP of smoking cessation at weeks I-2, 3-6, 7-10, and at or beyond week II were $51.7 \%$ (387/749), 59.6\% (I740/2922), 73.3\% (III4/I520), and 77.0\% (III6/I449), respectively (p for trend = 0.023). Using the total study subject number of 3700 as the denominator, the 7 -day PP of smoking cessation at the four visits was $10.5 \%, 47.0 \%, 30.1 \%$, and $30.2 \%$, respectively $(P$ for trend $=0.448)$. 
Table 4 Smoking Cessation Rate by Baseline Characteristics and Treatment Pattern

\begin{tabular}{|c|c|c|c|c|c|}
\hline & & $\begin{array}{l}\text { Smoking Cessation Rate \% } \\
\text { (N) }\end{array}$ & $\begin{array}{l}95 \% \text { Cl (Lower, } \\
\text { Upper) }\end{array}$ & Total $\mathbf{N}$ & p value ${ }^{b}$ \\
\hline \multirow[t]{2}{*}{ Sex } & Male & $67.9(2280)$ & $(66.3,69.4)$ & 3360 & \multirow[t]{2}{*}{0.800} \\
\hline & Female & $68.5(233)$ & $(63.6,73.5)$ & 340 & \\
\hline \multirow[t]{6}{*}{ Age (years) } & $<30$ & $70.5(105)$ & $(63.1,77.8)$ & 149 & \multirow[t]{6}{*}{0.019} \\
\hline & $30-39$ & 71.4 (459) & $(67.9,74.9)$ & 643 & \\
\hline & $40-49$ & 69.7 (772) & $(67.0,72.4)$ & 1107 & \\
\hline & $50-59$ & $66.8(7 \mid I)$ & $(63.9,69.6)$ & 1065 & \\
\hline & $60-69$ & $63.2(359)$ & $(59.2,67.2)$ & 568 & \\
\hline & $\geq 70$ & $63.7(107)$ & $(56.4,71.0)$ & 168 & \\
\hline \multirow{3}{*}{$\begin{array}{l}\text { Alcohol use (number of drinks per week) at } \\
\text { baseline }\end{array}$} & None & $65.0(474)$ & $(61.6,68.5)$ & 729 & \multirow[t]{3}{*}{$<0.001$} \\
\hline & $1-2$ & 70.7 (155I) & $(68.8,72.6)$ & 2193 & \\
\hline & $\geq 3$ & $62.7(487)$ & $(59.3,66.1)$ & 777 & \\
\hline \multirow[t]{4}{*}{ Age at smoking initiation ${ }^{\mathrm{a}}$ (years) } & $<15$ & $62.8(76)$ & $(54.2,71.4)$ & 121 & \multirow[t]{4}{*}{0.085} \\
\hline & $15-20$ & $66.7(1058)$ & $(64.4,69.0)$ & 1587 & \\
\hline & $21-30$ & $69.6(1316)$ & $(67.5,71.7)$ & 1891 & \\
\hline & Others & $62.0(62)$ & $(52.5,71.5)$ & 100 & \\
\hline \multirow[t]{4}{*}{ Average smoking amount per day (packs) ${ }^{\mathrm{a}}$} & $<1$ & $72.7(870)$ & $(70.2,75.2)$ & 1197 & \multirow[t]{4}{*}{$<0.001$} \\
\hline & $1-2$ & $65.8(1388)$ & $(63.8,67.9)$ & 2108 & \\
\hline & $2-3$ & 64.7 (244) & $(59.9,69.6)$ & 377 & \\
\hline & Others & $58.8(10)$ & $(35.4,82.2)$ & 17 & \\
\hline \multirow[t]{4}{*}{ Number of attempts to quit smoking } & None & $68.4(663)$ & $(65.5,71.4)$ & 969 & \multirow[t]{4}{*}{0.050} \\
\hline & Once & $71.7(521)$ & $(68.4,74.9)$ & 727 & \\
\hline & $2-4$ times & $67.0(943)$ & $(64.5,69.4)$ & 1408 & \\
\hline & $\geq 5$ times & $64.9(383)$ & $(61.1,68.8)$ & 590 & \\
\hline \multirow[t]{2}{*}{ Allergy history } & No & $68.2(2489)$ & $(66.6,69.7)$ & 3652 & \multirow[t]{2}{*}{0.007} \\
\hline & Yes & $50.0(24)$ & $(35.9,64.2)$ & 48 & \\
\hline \multirow[t]{9}{*}{ Comorbidity } & No & $72.6(1517)$ & $(70.7,74.5)$ & 2090 & \multirow[t]{2}{*}{$<0.001$} \\
\hline & Yes & 61.9 (996) & $(59.5,64.2)$ & 1610 & \\
\hline & $\mathrm{CHD}$ & $50.0(60)$ & $(41.1,59.0)$ & 120 & \\
\hline & Diabetes mellitus & $57.5(238)$ & $(52.7,62.3)$ & 414 & \\
\hline & $\begin{array}{l}\text { COPD and other pulmonary } \\
\text { disease }\end{array}$ & $57.5(287)$ & $(53.2,61.9)$ & 499 & \\
\hline & Hypertension & $63.9(455)$ & $(60.4,67.4)$ & 712 & \\
\hline & Dyslipidemia & $60.9(201)$ & $(55.6,66.2)$ & 330 & \\
\hline & Stroke or TIA & $68.6(24)$ & $(53.2,84.0)$ & 35 & \\
\hline & Others & $60.4(209)$ & $(55.3,65.6)$ & 346 & \\
\hline
\end{tabular}

(Continued) 
Table 4 (Continued).

\begin{tabular}{|c|c|c|c|c|c|}
\hline & & $\begin{array}{l}\text { Smoking Cessation Rate \% } \\
\text { (N) }\end{array}$ & $\begin{array}{l}95 \% \mathrm{Cl} \text { (Lower, } \\
\text { Upper) }\end{array}$ & Total $\mathbf{N}$ & p value ${ }^{b}$ \\
\hline \multirow[t]{2}{*}{ Concomitant medication } & No & $72.5(1606)$ & $(70.7,74.4)$ & 2215 & \multirow[t]{2}{*}{$<0.001$} \\
\hline & Yes & $61.1(907)$ & $(58.6,63.6)$ & 1485 & \\
\hline \multirow{3}{*}{$\begin{array}{l}\text { Total administration period of varenicline } \\
\text { (weeks) }\end{array}$} & $<4$ & $55.3(474)$ & $(52.0,58.6)$ & 857 & \multirow[t]{3}{*}{$<0.0001$} \\
\hline & $4-<8$ & $60.4(7 \mid 8)$ & $(57.66,63.22)$ & 1188 & \\
\hline & $\geq 8$ & 79.8 (I32I) & $(77.9,81.8)$ & 1655 & \\
\hline \multirow[t]{4}{*}{ Mean daily dose of varenicline (mg) } & $0.5-<1.0$ & $49.3(113)$ & $(42.9,55.8)$ & 229 & \multirow[t]{4}{*}{$<0.0001$} \\
\hline & $1.0-<1.5$ & $60.6(260)$ & $(56.0,65.2)$ & 429 & \\
\hline & $1.5-<2.0$ & $70.6(2096)$ & $(69.0,72.3)$ & 2967 & \\
\hline & $\geq 2.0$ & $58.7(44)$ & $(47.5,69.8)$ & 75 & \\
\hline \multicolumn{2}{|l|}{ Total } & $67.9(25 \mid 3)$ & $(66.4,69.4)$ & 3700 & \\
\hline
\end{tabular}

Notes: "Among "Others" for age at smoking initiation, 83 subjects started smoking after 30 years of age, while 17 subjects provided no information. Among "Others" for average smoking amount per day, 16 subjects smoked three packs or more a day on average, while one subject provided no information; ${ }^{\mathrm{b}} \mathrm{p}$ value was calculated using Chisquare test.

Abbreviations: $\mathrm{Cl}$, confidence interval; $\mathrm{N}$, number of study subjects; $\mathrm{CHD}$, congestive heart disease; COPD, chronic obstructive pulmonary disease; TIA, transient ischemic attack.

risk of neuropsychiatric AEs, including suicide, suicidal ideation, and depression. ${ }^{14}$ A network meta-analysis study on varenicline reported no increase in the risk of cardiovascular disease events with varenicline. ${ }^{21}$

According to our study results, there were no new or significant safety concerns for Korean smokers taking varenicline. Of the 3719 subjects, 346 (9.3\%) experienced 471 AEs, most of which were mild (358/471 events; $76.0 \%$ ). Nausea was the most common AE and developed in 5.1\% of subjects, and most reported cases were of mild or moderate intensity. Overall, 68 subjects discontinued the study due to AEs, and four experienced seven SAEs, four of which were considered related to the study drug. Further, 78 subjects reported psychiatric AEs, most of which were sleep disturbances, and no severe cases were observed. No major AEs directly related to cardiovascular disease were reported

Table 5 Smoking Cessation and Continuation at Last Visit by Study Completion and Drop Out Reasons

\begin{tabular}{|l|l|l|l|l|l|}
\hline \multicolumn{2}{|l|}{} & \multicolumn{2}{|l|}{$\begin{array}{l}\text { Smoking Cessation at Last Visit, } \\
\text { n (\%) }\end{array}$} & $\begin{array}{l}\text { Continued Smoking at } \\
\text { Last Visit, n (\%) }\end{array}$ & Total, n (\%) \\
\hline $\begin{array}{l}\text { Study completion at I2 } \\
\text { weeks }\end{array}$ & Yes & $971(83.4)$ & $194(16.6)$ & $1165(31.5)$ \\
\cline { 2 - 5 } & No & $1542(60.8)$ & $993(39.2)$ & $2535(68.5)$ \\
\cline { 2 - 5 } & Total & $2513(67.9)$ & $1187(32.1)$ & $3700(100)$ \\
\hline Reasons for study discontinuation & Smoking cessation group & Continued smoking group & \\
\hline Lost to follow up & $578(37.5)$ & $378(38.1)$ & $956(37.7)$ \\
\hline Smoking cessation & $931(60.4)$ & $7(0.7)$ & $938(37.0)$ \\
\hline Smoking continued or increased & $3(0.2)$ & $552(55.6)$ & $555(21.9)$ \\
\hline Adverse events & $25(1.6)$ & $43(4.3)$ & $68(2.7)$ \\
\hline Others or missing data & $5(0.3)$ & $13(1.3)$ & $18(0.7)$ \\
\hline Total & $1542(100)$ & $993(100)$ & $2535(100)$ \\
\hline
\end{tabular}

Abbreviation: NA, not applicable. 
except for one case of cerebral artery stenosis, which was considered as unrelated to varenicline by the investigator. These findings agreed with previous studies that showed no increase in the risk of psychiatric problems and serious cardiovascular AEs in the Asian populations. ${ }^{22}$ The malignancy was not observed even in long-term users of this study, except for one subject who had administered varenicline for about 2 months reporting aggravation of lung neoplasm; however, the investigator evaluated this as unrelated to the study drug. In previous studies of long-term varenicline use up to 52 weeks of treatment, cancer was not reported. ${ }^{23,24}$ AEs may have been somewhat underreported because the study was conducted in a real-world setting and the follow-up period was short; however, varenicline tend not to be used in long-term, and the AE profile and severity distribution were similar to the preceding studies. ${ }^{22}$

No other sociodemographic factors were associated with an increase in AE incidence except for allergy history or taking other medications. Presently, there are no reported drug interactions with varenicline, and it is not known to interact with cytochrome P450 enzymes. ${ }^{12}$ Further studies may be necessary to identify the possibility of interactions between varenicline and other drugs. A higher percentage of subjects reported AEs in the group with a lower mean daily dose of varenicline than the group taking a higher mean daily dose $(p=0.027)$, suggesting that AEs occur more frequently during the early titration period when the medication is first introduced into the body.

In line with previous reports, varenicline was effective for smoking cessation in this study. The 7-day PP of smoking cessation at the last observed study visit was $67.9 \%$, which was high compared with other interventional or observational studies, ${ }^{9,10}$ suggesting that subjects with high will to quit made follow-up visits. When estimated in subjects who returned to clinics for each visit, 7-day PP increased from baseline to week 12 (Figure 2), supporting the trend of decreasing smoking rate after varenicline treatment in real-world clinical practice. A significantly higher cessation rate in subjects who completed the study $(83.4 \%$ vs $60.8 \%)$ supports this finding. When subjects with missing visits were included in the calculation, the 7-day PP at week 12 was 30.2\%, approaching the 7-day PP results from randomized controlled studies that enrolled smokers not yet ready to quit. ${ }^{25,26}$

According to a multi-center prospective observational study conducted in China, India, the Philippines, and South Korea, $20.3 \%$ Korean participants maintained smoking cessation after 12 -week varenicline treatment. ${ }^{10}$ In this study, only $25 \%$ Korean participants completed the study; therefore, a large portion of subjects were unknown of their smoking status. This can be partially explained by the fact that varenicline is relatively expensive in South Korea and not reimbursed by the National Health Insurance System as well as the varenicline label being mandated to carry "black box warning" about the risk of serious neuropsychiatric events by the FDA in 2009; however, as shown in our study, early achievement of smoking cessation could have been a reason for high discontinuation rate, that is, many of those who successfully quit may have discontinued treatment early.

Age, frequency of alcohol intake, average number of cigarettes per day, number of previous attempts to quit, allergy history, comorbidity, and concomitant medications were significantly associated with smoking cessation in this study. Younger, lighter smokers and less frequent quit attempters were more likely to stop smoking than older, heavier smokers and more frequent attempters, which may have resulted from difference in nicotine dependence. The smoking cessation rate was significantly lower in subjects who consumed alcohol more than thrice per week than less frequent drinkers, and previous studies confirmed that drinking related to social factors increased the desire to smoke. ${ }^{27,28}$ To increase the chances of quitting smoking, it is necessary to encourage smokers to reduce or avoid alcohol consumption. Smokers with allergy history, comorbidities, and concomitant medications may have experienced more AEs, leading to less compliance and lower quit rate. It is important to note that subjects with underlying congestive heart disease, diabetes mellitus, and pulmonary diseases including chronic obstructive pulmonary disease, who already are suffering from smoking-related diseases, showed relatively low smoking cessation rate at $50 \mathrm{~s} \%$. These patients may likely be chronic heavy smokers and need especially intensive care. Previous and recent works have shown consistent benefit of varenicline in various patient groups for smoking cessation and the following clinical outcomes. ${ }^{29-33}$

This study has several limitations. First, it is likely that AEs were underreported, considering non-interventional study, wherein subjects were not required to make predetermined study visits. Second, smoking cessation status was selfreported without any biological evidence like the carbon monoxide breath test result, which may lead to an overestimation of effectiveness. Third, the dropout rate was high, which may also have resulted in an underestimation of AE incidence and overestimation of the smoking cessation rate. When the baseline characteristics were compared between 
the study subjects who completed the study and who discontinued the study, the characteristics were all similar, except the proportion of concomitant medication users which was higher in completers (42.4\%) than in discontinuers (38.8\%) $(\mathrm{p}=0.04)$ (Supplementary Table 3$)$. However, this difference in concomitant medication usage may not affect the clinical relevance of the overall study results. Also, high dropout rates from smoking cessation programs are common in realworld clinical practice; thus, our study results could be more generalizable. Also, many subjects who did not complete our study reported achieving smoking cessation before the end of the 12-week treatment course, which may mitigate the likelihood of overestimating the quit rate. Lastly, the 7-day PP of smoking cessation may provide only a rough indication of treatment effectiveness.

Despite these limitations, our study significantly contributes to the safety of varenicline in the Asian population. Although previous studies have evidenced the efficacy of varenicline in terms of smoking cessation rates, ${ }^{34-36}$ varenicline has not been sufficiently used for smoking cessation in South Korea, and there is still room for improvement in patient compliance to clinical guidelines. ${ }^{37}$ Therefore, it is necessary to continue to support varenicline use as a first-line drug for smoking cessation in all clinical settings. ${ }^{28,38}$

\section{Conclusion}

Taken together, varenicline was well tolerated and effective for smoking cessation in real-world clinical practice. The most common AEs in Korean smokers were gastrointestinal-related symptoms, and the AE incidence almost doubled in study subjects with a history of allergy, comorbidity, or concomitant medications. Varenicline was effective with a high smoking cessation rate that was associated with younger age, less frequent drinking, smoking less than one pack per day, few attempts to quit smoking, and no use of concomitant medications.

\section{Data Sharing Statement}

According to the informed consent form used for this research, participants did not agree for their individual data to be shared publicly. However, the authors confirm that the data supporting the findings of this study are available within the article and its supplementary materials.

\section{Acknowledgments}

All investigators who participated in this study are listed in the Supplementary Table 1. We would like to express our special gratitude to Song-Hwa Choi for conducting a constructive review of the manuscript.

\section{Funding}

This research was sponsored by Pfizer; however, the authors received no payment for manuscript development.

\section{Disclosure}

Seung Won $\mathrm{Ra}$ is a paid consultant for Pfizer Pharmaceuticals Korea Ltd. and has contributed to this study in investigation, interpretation of analysis results, writing original draft manuscript, and review and editing of manuscript.

Ji Soo Shin is a full-time employee of, and holds stock in, Pfizer Inc. and has contributed to this study in interpretation of analysis results, writing original draft manuscript, review and editing of manuscript, and project administration. The authors report no other conflicts of interest in this work.

The abstract of this paper was presented at the American Thoracic Society 2019 International Conference in Dallas, Texas, USA, in May 2019. The poster's abstract was published in "Poster Abstracts" as follows: https://conference. thoracic.org/program/resources/2019/virtual-final-program/343/

\section{References}

1. Iribarren C, Tekawa IS, Sidney S, Friedman GD. Effect of cigar smoking on the risk of cardiovascular disease, chronic obstructive pulmonary disease, and cancer in men. $N$ Engl J Med. 1999;340(23):1773-1780. doi:10.1056/NEJM199906103402301

2. Reitsma MB, Fullman N, Ng M, et al. Smoking prevalence and attributable disease burden in 195 countries and territories, 1990-2015: a systematic analysis from the Global Burden of Disease Study 2015. Lancet. 2017;389(10082):1885-1906. doi:10.1016/S0140-6736(17)30819-X 
3. Hong JW, Noh JH, Kim DJ. The prevalence of and factors associated with urinary cotinine-verified smoking in Korean adults: the 2008-2011 Korea National Health and Nutrition Examination Survey. PLoS One. 2018;13(6):e0198814. doi:10.1371/journal.pone.0198814

4. Prochaska JJ, Benowitz NL. The past, present, and future of nicotine addiction therapy. Annu Rev Med. 2016;67:467-486. doi:10.1146/annurevmed-111314-033712

5. Tonstad S, Tonnesen P, Hajek P, Williams KE, Billing CB, Reeves KR; for Varenicline Phase 3 Study Group. Effect of maintenance therapy with varenicline on smoking cessation: a randomized controlled trial. JAMA. 2006;296(1):64-71. doi:10.1001/jama.296.1.64

6. Treating tobacco use and dependence: 2008 update: clinical practice guideline. Rockville, MD: US Department of Health and Human Services, Public Health Service. Available from: https://www.ahrq.gov/professionals/clinicians-providers/guidelines-recommendations/tobacco/index.html. Accessed October 6, 2021.

7. Fagerström K, Hughes J. Varenicline in the treatment of tobacco dependence. Neuropsychiatr Dis Treat. 2008;4(2):353-363. doi:10.2147/NDT. S927

8. Garrison GD, Dugan SE. Varenicline: a first-line treatment option for smoking cessation. Clin Ther. 2009;31(3):463-491. doi:10.1016/j. clinthera.2009.03.021

9. Fagerström K, Nakamura M, Cho HJ, et al. Varenicline treatment for smoking cessation in Asian populations: a pooled analysis of placebo-controlled trials conducted in six Asian countries. Curr Med Res Opin. 2010;26(9):2165-2173. doi:10.1185/03007995.2010.505130

10. Wang C, Cho B, Xiao D, Wajsbrot D, Park PW. Effectiveness and safety of varenicline as an aid to smoking cessation: results of an inter-Asian observational study in real-world clinical practice. Int J Clin Pract. 2013;67(5):469-476. doi:10.1111/ijcp.12121

11. Anthenelli RM, Benowitz NL, West R, et al. Neuropsychiatric safety and efficacy of varenicline, bupropion, and nicotine patch in smokers with and without psychiatric disorders (EAGLES): a double-blind, randomised, placebo-controlled clinical trial. Lancet. 2016;387(10037):2507-2520. doi:10.1016/S0140-6736(16)30272-0

12. Jordan CJ, Xi ZX. Discovery and development of varenicline for smoking cessation. Expert Opin Drug Discov. 2018;13(7):671-683. doi:10.1080/ 17460441.2018 .1458090

13. Pasternak B, Svanstrom H, Hviid A. Use of varenicline versus bupropion and risk of psychiatric adverse events. Addiction. 2013;108 (7):1336-1343. doi:10.1111/add.12165

14. Thomas KH, Martin RM, Knipe DW, Higgins JP, Gunnell D. Risk of neuropsychiatric adverse events associated with varenicline: systematic review and meta-analysis. $B M J .2015 ; 350: \mathrm{h} 1109$. doi:10.1136/bmj.h1109

15. Vlahović-Palčevski V, Mentzer D. Postmarketing surveillance. Handb Exp Pharmacol. 2011;205:339-351.

16. Food and Drug Administration FDA Drug Safety Communication. Safety review update of Chantix (varenicline) and risk of neuropsychiatric adverse events. Available from: http://www.fda.gov/Drugs/DrugSafety/ucm276737.htm. Accessed October 6, 2021.

17. Food and Drug Administration FDA Drug Safety Communication. Safety review update of Chantix (varenicline) and risk of cardiovascular adverse events. Available from: http://www.fda.gov/Drugs/DrugSafety/ucm330367.htm. Accessed October 6, 2021.

18. Thomas KH, Martin RM, Davies NM, Metcalfe C, Windmeijer F, Gunnell D. Smoking cessation treatment and risk of depression, suicide, and self harm in the Clinical Practice Research Datalink: prospective cohort study. BMJ. 2013;347:f5704. doi:10.1136/bmj.f5704

19. Singh S, Loke YK, Spangler JG, Furberg CD. Risk of serious adverse cardiovascular events associated with varenicline: a systematic review and meta-analysis. CMAJ. 2011;183(12):1359-1366. doi:10.1503/cmaj.110218

20. Prochaska JJ, Hilton JF. Risk of cardiovascular serious adverse events associated with varenicline use for tobacco cessation: systematic review and meta-analysis. BMJ. 2012;344:e2856. doi:10.1136/bmj.e2856

21. Mills EJ, Thorlund K, Eapen S, Wu P, Prochaska JJ. Cardiovascular events associated with smoking cessation pharmacotherapies: a network meta-analysis. Circulation. 2014;129(1):28-41. doi:10.1161/CIRCULATIONAHA.113.003961

22. Xiao D, Chu S, Wang C. Smoking cessation in Asians: focus on varenicline. Patient Prefer Adherence. 2015;9:579-584. doi:10.2147/PPA.S60785

23. Williams KE, Reeves KR, Billing CB, Pennington AM, Gong J. A double-blind study evaluating the long-term safety of varenicline for smoking cessation. Curr Med Res Opin. 2007;23(4):793-801. doi:10.1185/030079907X182185

24. Evins AE, Cather C, Pratt SA, et al. Maintenance treatment with varenicline for smoking cessation in patients with schizophrenia and bipolar disorder: a randomized clinical trial. JAMA. 2014;311(2):145-154. doi:10.1001/jama.2013.285113

25. Hughes JR, Rennard SI, Fingar JR, Talbot SK, Callas PW, Fagerstrom KO. Efficacy of varenicline to prompt quit attempts in smokers not currently trying to quit: a randomized placebo-controlled trial. Nicotine Tob Res. 2011;13(10):955-964. doi:10.1093/ntr/ntr103

26. Ebbert JO, Hughes JR, West RJ, et al. Effect of varenicline on smoking cessation through smoking reduction: a randomized clinical trial. JAMA. 2015;313(7):687-694. doi:10.1001/jama.2015.280

27. Friend KB, Pagano ME. Smoking cessation and alcohol consumption in individuals in treatment for alcohol use disorders. J Addict Dis. 2005;24 (2):61-75. doi:10.1300/J069v24n02_06

28. Pyo J, Ock M, Lee JW, Han YJ, Jo MW, Lee JA. Experience of smoking cessation treatment program in Korea: results from a qualitative study. Health Policy Manag. 2017;27(4):284-295.

29. Ioakeimidis N, Georgakopoulos C, Emmanouil E, et al. Effect of smoking cessation with varenicline on blood pressure control in hypertensive patients. Eur Heart J. 2020;41(Suppl 2):ehaa946.3019. doi:10.1093/ehjci/ehaa946.3019

30. Pezzuto A, Carico E. Effectiveness of smoking cessation in smokers with COPD and nocturnal oxygen desaturation: functional analysis. Clin Respir J. 2020;14(1):29-34. doi:10.1111/crj.13096

31. Pezzuto A, D'Ascanio M, Grieco A, Ricci A. Functional benefit of smoking cessation in severe COPD patients undergoing bronchial valve implantation. Eur J Intern Med. 2019;68:55-59. doi:10.1016/j.ejim.2019.07.032

32. Tashkin DP, Murray RP. Smoking cessation in chronic obstructive pulmonary disease. Respir Med. 2009;103(7):963-974. doi:10.1016/j. rmed.2009.02.013

33. Tonstad S, Lawrence D. Varenicline in smokers with diabetes: a pooled analysis of 15 randomized, placebo-controlled studies of varenicline. $J$ Diabetes Investig. 2017;8(1):93-100. doi:10.1111/jdi.12543

34. Gonzales D, Rennard SI, Nides M, et al. Varenicline, an $\alpha 4 \beta 2$ nicotinic acetylcholine receptor partial agonist, vs sustained-release bupropion and placebo for smoking cessation: a randomized controlled trial. JAMA. 2006;296(1):47-55. doi:10.1001/jama.296.1.47 
35. Jorenby DE, Hays JT, Rigotti NA, et al.; for Varenicline Phase 3 Study Group. Efficacy of varenicline, an $\alpha 4 \beta 2$ nicotinic acetylcholine receptor partial agonist, vs placebo or sustained-release bupropion for smoking cessation: a randomized controlled trial. JAMA. 2006;296(1):56-63. doi:10.1001/jama.296.1.56

36. Aubin HJ, Bobak A, Britton JR, et al. Varenicline versus transdermal nicotine patch for smoking cessation: results from a randomised open-label trial. Thorax. 2008;63(8):717-724. doi:10.1136/thx.2007.090647

37. Liberman JN, Lichtenfeld MJ, Galaznik A, et al. Adherence to varenicline and associated smoking cessation in a community-based patient setting. J Manag Care Pharm. 2013;19(2):125-131. doi:10.18553/jmcp.2013.19.2.125

38. Kim H, Oh JK, Lim MK, Jeong BY, Yun EH, Park EY. The national "smoking cessation clinics" program in the Republic of Korea: socioeconomic status and age matter. Asian Pac J Cancer Prev. 2013;14(11):6919-6924. doi:10.7314/APJCP.2013.14.11.6919

\section{Publish your work in this journal}

Patient Preference and Adherence is an international, peer-reviewed, open access journal that focusing on the growing importance of patient preference and adherence throughout the therapeutic continuum. Patient satisfaction, acceptability, quality of life, compliance, persistence and their role in developing new therapeutic modalities and compounds to optimize clinical outcomes for existing disease states are major areas of interest for the journal. This journal has been accepted for indexing on PubMed Central. The manuscript management system is completely online and includes a very quick and fair peer-review system, which is all easy to use. Visit http://www.dovepress.com/testimonials.php to read real quotes from published authors.

Submit your manuscript here: https://www.dovepress.com/patient-preference-and-adherence-journal 